\title{
CONSUMO DE ALIMENTOS FORTIFICADOS EN ESTUDIANTES SECUNDARIOS DE LA REGIÓN METROPOLITANA DE CHILE
}

\section{FORTIFIED FOOD CONSUMPTION IN HIGH SCHOOL STUDENTS FROM THE METROPOLITAN REGION OF CHILE}

\author{
Samuel Durán A. (1), Alejandra Freixas S. (1), Josseline Saavedra M. (2), \\ Rodrigo Maureira L. (2), Daniela Berrios O. (2), María Cristina Gaete V. (1) \\ (1) Universidad Autónoma de Chile. Santiago, Chile. \\ (2) Carrera de Nutrición y Dietética. Universidad Autónoma de Chile. Santiago, Chile.
}

\begin{abstract}
Introduction: Vitamins are nutrients that have multiple benefits for our body which are used to enrich foods to increase nutritional intake. Objective: To determine whether the intake of fortified foods exceeded dietary recommendations (RDA) and tolerable upper intake level (UL). Methods: 213 fortified foods on the market were identified. An alimentary survey was performed using a photographic set of 298 foods in high school males and females students from Metropolitan Region. The intake of nutrients was calculated and the fortified vitamins on foods were added. Results: Men exceeded recommended intake for all vitamins, whereas women have a deficit in B12 Vitamin. Vitamins whose intake exceeds the UL are, folate, $27.5 \%, B 3$ vitamin $16.4 \%$, A vitamin $7.8 \%$ and B6 vitamin 1.6\%. Conclusion: All tested vitamins exceeded recommendations except for B1 vitamins in women; folate; B3, B6 and A vitamin exceed the $U L$.
\end{abstract}

Key words: Vitamins, dietary survey, $U L, R D A$.

Este trabajo fue recibido el 26 de Octubre de 2011 y aceptado para ser publicado el 12 de Marzo de 2012.

\section{INTRODUCCIÓN}

Las vitaminas forman parte de los nutrientes orgánicos, necesarios en pequeñas cantidades (micronutrientes) para diversas funciones bioquímicas, que no pueden ser sintetizadas por el organismo, por lo que se obtienen a través de la dieta, con el fin de mantener la integridad metabólica normal $(1,2)$. Una dieta variada que cumple con las directrices dietéticas basadas en los alimentos cubre las necesidades de la mayoría de las vitaminas y los minerales en la población (3).

Sin embargo, muchas personas tienen dietas con bajo contenido de uno o más nutrientes, especialmente adolescentes, ancianos, embarazadas, fumadores, bebedores y en general, las personas que siguen regímenes dietéticos deficientes precisan de un mayor aporte de estas sustancias, sin valor energético pero dotadas de una gran actividad biológica (4-8). Una alternativa a una ingesta deficiente en vitaminas es la fortificación de ellas en los alimentos.
La fortificación se ha definido como la adición de uno o más nutrientes a un alimento a fin de mejorar su calidad, con el objeto de reducir o controlar una carencia de nutrientes, en una concentración de un $10 \%$ o más de la Dosis Diaria de Referencia (DDR) por porción de consumo habitual para un nutriente en particular (9).

La adición de nutrientes a los alimentos, ya sea por enriquecimiento (en sustitución de nutrientes que se pierden en el proceso) o de la fortificación (la adición de nutrientes en los niveles superiores a los que se producen naturalmente en el alimento), mejora los niveles de uno o más nutrientes de ciertos alimentos de consumo masivo, lo que aumenta la ingesta a niveles más deseables (10). La mayoría de los productos como cereales enriquecidos y una variedad de otros alimentos están fortificados. Por ejemplo, el pan se ha enriquecido con tiamina, niacina, riboflavina y hierro, la mayoría de los cereales están fortificados con hierro y vitaminas del complejo B, incluyendo ácido fólico, y la mayoría de la 
leche está fortificada con vitamina D (10). Sin embargo es necesario evaluar las ventajas y desventajas de la fortificación en cada circunstancia. En Europa, los alimentos fortificados no contribuyen de forma significativa a la ingesta de nutrientes (11), en el caso de Estados Unidos existe poca información sobre ingesta de micronutrientes en alimentos fortificados a excepción del folato (12). El objetivo del presente estudio es analizar la ingesta de alimentos fortificados con vitaminas en estudiantes secundarios de Región Metropolitana y compararla con las Recomendaciones y la UL (nivel máximo de ingesta tolerable).

\section{SUJETOS Y MÉTODOS}

Se realizó un estudio descriptivo a 298 estudiantes secundarios de la Región Metropolitana.

El tamaño de la muestral se calculó a partir del estudio de Fulgoni (10) con un intervalo de confianza del $95 \%$, una potencia de un $90 \%$ y una precisión calculada como media observada- valor recomendado (RDA) para cada vitamina.

Todos los participantes dieron su asentimiento por escrito y sus padres firmaron el consentimiento. El estudio fue aprobado por el Comité de Ética de la Universidad Autónoma de Chile.

Se identificaron 213 alimentos fortificados del mercado (cereales de desayuno, galletas, arroz, zumos y jugos en polvo, lácteos, margarinas y bebidas energéticas), se registró el aporte de cada vitamina contenida del alimento. Se tomaron fotografías en los supermercados de los alimentos. Posteriormente se les realizó una encuesta de tendencia de consumo a los estudiantes, para lo cual se creó paneles fotográficos con los diferentes alimentos disponibles en el mercado, que permitieron al encuestador y encuestado precisar de manera más exacta los registros.

Posteriormente se realizó el cálculo de ingesta de nutrientes utilizando las tablas de composición química de alimentos $(13,14)$. Al resultado anterior se sumó la cantidad de las vitaminas fortificadas contenida en los alimentos cuando correspondía, obteniéndose 3 valores distintos: 1. Aporte de vitaminas según tabla de composición, 2. Aporte de vitaminas en alimentos fortificados, 3 . Ingesta de total de vitaminas (suma de las 2 anteriores).

Con la Dosis Diaria Recomendada (DDR) o también denominada cantidad diaria recomendada (con sus siglas en inglés como RDA) se estimó si existe un exceso o un déficit en el consumo de vitaminas de los estudiantes y si estos sobrepasaban la UL, la información utilizada fue obtenida a partir de Dietary Guidelines for Americans, 2010 (15).

\section{ANÁLISIS ESTADÍSTICO}

Los datos fueron procesados en una planilla Excel y en el programa estadístico SPSS 19.0. Para evaluar la normalidad de las variables continuas (edad, peso, talla, ingesta) se utilizó la prueba de bondad de Shapiro Wilk's. Para las variables normales se utilizó la prueba estadística Test t de Student y para las no normales Mann-Whitney U. En todos los casos se consideró un valor de $\mathrm{p}<0,05$.

\section{RESULTADOS}

De los estudiantes secundarios encuestados el $68,9 \%$ consumen alimentos fortificados con vitamina A, $69,3 \%$ con vitamina B1, 84,7\% con vitamina B2, $75.6 \%$ con vitamina $B 3,72,7 \%$ con vitamina $B 6,26,1 \%$ con vitamina $\mathrm{B} 9,70,5 \%$ con vitamina $\mathrm{B} 12,54,4 \%$ con ácido pantoténico, $85,6 \%$ con vitamina $\mathrm{C}$ y $51,7 \%$ con vitamina E. El consumo promedio de alimentos fortificados fue de 2,6 alimentos/día por estudiante. Entre los alimentos fortificados de mayor consumo podemos nombrar la leche, pasta, arroz, cereales de desayuno y jugos, y los menos consumidos son las bebidas energéticas, margarinas y barras de cereal.

Al comparar por sexo las diferencias se muestran en la tabla 1. Siendo mayor el consumo en la mayoría de las vitaminas en los hombres.

En el caso de los estudiantes varones (gráfico 1) la ingesta de vitaminas obtenida sólo de la tabla de composición, todos superan la recomendación en todas las vitaminas evaluadas (vitamina A 201\%, B1 237\%, B2 $170 \%$, B3 162\%, B6 155\%, folato $213 \%$, ácido pantoténico $111 \%$, B12 104\%, C 183\%), en el caso de las mujeres (gráfico 2) la vitaminas B12 y ácido pantoténico están por debajo de las recomendaciones, el resto están sobrepasadas (Vitamina A 158\%, B1 172\%, B2 155\%, B3 106\%, B6 119\%, folato 150\%, B12 57\%, ácido pantoténico $76 \%$ y vitamina C $181 \%$ ).

Las vitaminas que vienen fortificados en los alimentos aportan entre un 9,8\% en el caso del folato y B12 y un $200 \%$ en el caso de la vitamina B2 en los hombres, en las mujeres el mínimo aporte se encuentra en la vitamina B 12 con un $12 \%$ de la recomendación en cambio el mayor aporte se encuentra con las vitaminas $\mathrm{C}$ y A con un 52 y $53 \%$ respectivamente.

Al sumar la ingesta de vitaminas obtenidas por la tabla de composición química de los alimentos y la fortificación en el caso de los hombres, todas las vitaminas superan las recomendaciones (vitamina A $242 \%$, B1 267\%, B2 370\%, B3 188\%, B6 190\%, folato 233\%, B12 113\%, ácido pantoténico 156\%, C 229\%). En el caso de las mujeres, todas las vitaminas a excepción de la B12 supera las recomendaciones (vitamina A $211 \%$, 
B1 210\%, B2 368\%, B3 133\%, B6 170\%, folato 171\%, B12 69\%, ácido pantoténico 105\%, C 233\%).

El porcentaje de población que supera la UL fue baja para la mayoría de las vitaminas debido a no considerar la fortificación de alimentos, al enumerar de mayor a menor $\%$ sobre la UL fue $20,5 \%$ para folato; $8,9 \%$ para $\mathrm{B} 3 ; 5,1 \%$ para vitamina $\mathrm{A}$. Al añadir al resultado anterior las vitaminas por alimentos fortificados, se incrementó el $\%$ de estudiantes que superan la UL y se agrega a la lista la vitamina B6, en orden decreciente podemos nombrar $27 \%$ para folato, $16,4 \% \mathrm{~B} 3,7,8 \%$ vitamina $\mathrm{A}$ y $1,3 \% \mathrm{~B} 6$, en el resto de las vitaminas ningún escolar superó la UL.

\section{DISCUSIÓN}

Las guías alimentarias recomiendan una ingesta de nutrientes a partir de fuentes alimentarias (15). Los alimentos contienen nutrientes de forma natural, muchas de las vitaminas que necesitamos las encontramos en una buena cantidad en alimentos como las carnes, lácteos, granos enteros, frutas y verduras. Otros alimentos que contienen naturalmente vitaminas se enriquecen o fortifican. Al igual que en Europa nuestros datos sugieren que el consumo de forma natural es el principal aporte de vitaminas (16), quizás con excepción de los folatos donde nuestra ingesta es superior al consumo europeo pero similar a los Estados Unidos (10). En Estados Unidos los alimentos presentan un mayor grado de enriquecimiento que en países europeos (16).

Estudios muestran que la fortificación de vitaminas como el ácido fólico y vitaminas del complejo B en alimentos incrementa los niveles de estas vitaminas tanto al evaluar por ingesta dietética como por los niveles de biomarcadores, lo que tendría un potencial efecto sobre la salud de los individuos (17). En Chile la harina se fortifica con tiamina $6,3 \mathrm{mg} / \mathrm{kg}$, Riboflavina $1,3 \mathrm{mg} / \mathrm{kg}$, Niacina $13 \mathrm{mg} / \mathrm{kg}$ y ácido fólico $1,8 \mathrm{mg} / \mathrm{kg}$ con un rango aceptable de 1,0 a 2,6 mg/kg9. El ácido fólico se le agrega a la harina de trigo en Chile desde enero del año 2000, con el fin de reducir el riesgo de defectos del tubo neural (18). Sin embargo, publicaciones sugieren que la ingesta excesiva de ácido fólico podría conferir un mayor riesgo de cáncer, en especial el cáncer colorrectal. La evidencia ha llegado en su mayoría de trabajos in vitro y los datos experimentales en animales, el folato presenta un "doble efecto" en células tumorales, lo que sugiere que a niveles bajos de folato, suplementos de ácido fólico disminuye el riesgo de cáncer, y en altos niveles de exposición en el contex to de las células precancerosas,

\section{TABLA 1}

Comparación de la ingesta de macronutrientes y vitaminas entre estudiantes de distinto sexo.

\begin{tabular}{|llll|}
\hline Variables & Hombres (129) & Mujeres (166) & Valor p \\
\hline Edad (años) $^{\mathrm{a}}$ & $16,4 \pm 1,4$ & $16,9 \pm 1,5$ & 0,01 \\
Kcal $^{\mathrm{a}}$ & $3131,0 \pm 1607$ & $2325 \pm 939$ & 0,001 \\
Proteínas (g) $^{\mathrm{a}}$ & $117,0 \pm 68,5$ & $81,8 \pm 35,3$ & 0,001 \\
Lípidos (g) $^{\mathrm{a}}$ & $79,1 \pm 56,1$ & $62,6 \pm 34,1$ & 0,02 \\
Carbohidratos (g) $^{\mathrm{a}}$ & $485,7 \pm 242$ & $357 \pm 155$ & 0,001 \\
Vitamina A (ugER) $^{\mathrm{b}}$ & $1012,8(539,1-1693,9)$ & $928,9(448,0-1661,9)$ & 0,4 \\
Tiamina o B1 (mg) $^{\mathrm{b}}$ & $2,47(1,85-4,06)$ & $2,07(1,51-2,9)$ & 0,001 \\
Rivoflavina o B2 (mg) $^{\mathrm{b}}$ & $4,0(1,96-5,87)$ & $2,71(1,65-4,3)$ & 0,001 \\
Niacina o B3 (mgER) $^{\mathrm{b}}$ & $24,2(17,9-38,3)$ & $18,9(14,3-27,1)$ & 0,001 \\
Piridoxina o B6 (mg) $^{\mathrm{b}}$ & $1,84(1,33-3,1)$ & $1,56(1,10-2,34)$ & 0,001 \\
Ac.Fólico (ug) $^{\mathrm{b}}$ & $749,2(555,8-1164,3)$ & $604,2(403,4-860,9)$ & 0,001 \\
Cobalamina o B12 (ug) $^{\mathrm{b}}$ & $2,15(1,30-3,26)$ & $1,58(0,94-2,18)$ & 0,001 \\
Ac. Pantoténico (mg) $^{\mathrm{b}}$ & $5,19(3,9-8,2)$ & $4,08(3,3-5,88)$ & 0,001 \\
Vitamina C (mg) $^{\mathrm{b}}$ & $69,2(47,9-110,7)$ & $75,5(49,2-122,0)$ & 0,4 \\
Vitamina E (mgET) $^{\mathrm{b}}$ & $3,89(2,35-7,43)$ & $3,45(2,25-5,98)$ & 0,3 \\
\hline${ }^{a}$ Media (desviación estándar) $^{\mathrm{b}}$ Mediana (rango intercuartílico) & & & \\
\hline
\end{tabular}




\section{GRÁFICO 1}

Adecuación de la ingesta de vitaminas (\%) en varones según la RDA obtenido de la tabla de composición, fortificación de alimentos y consumo total

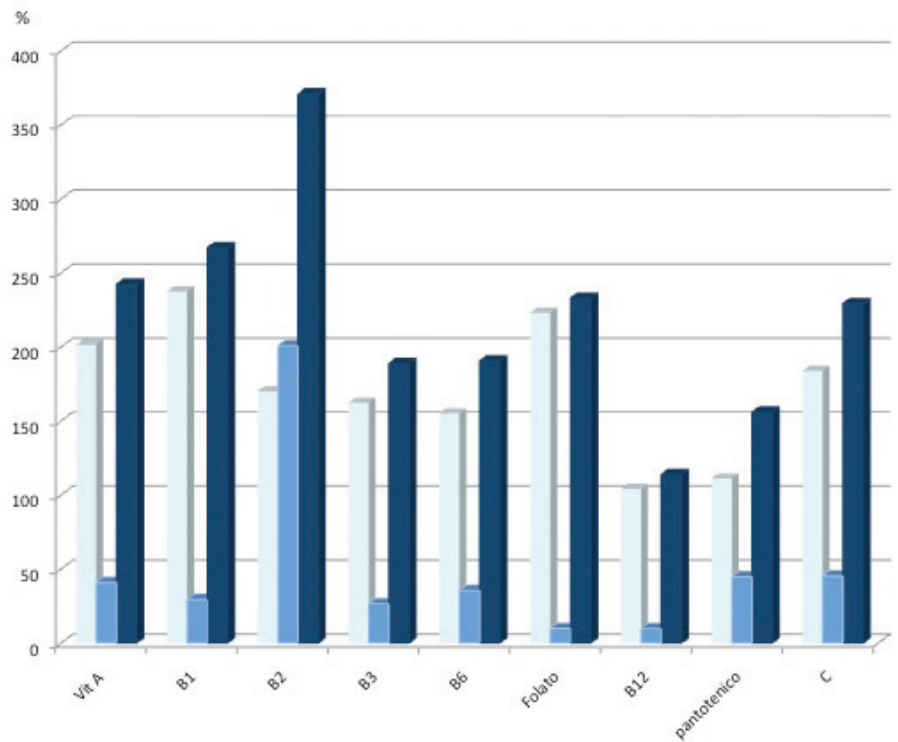

\section{GRÁFICO 2}

Adecuación de la ingesta de vitaminas (\%) en mujeres según la RDA obtenido de la tabla de composición, fortificación de alimentos y consumo total

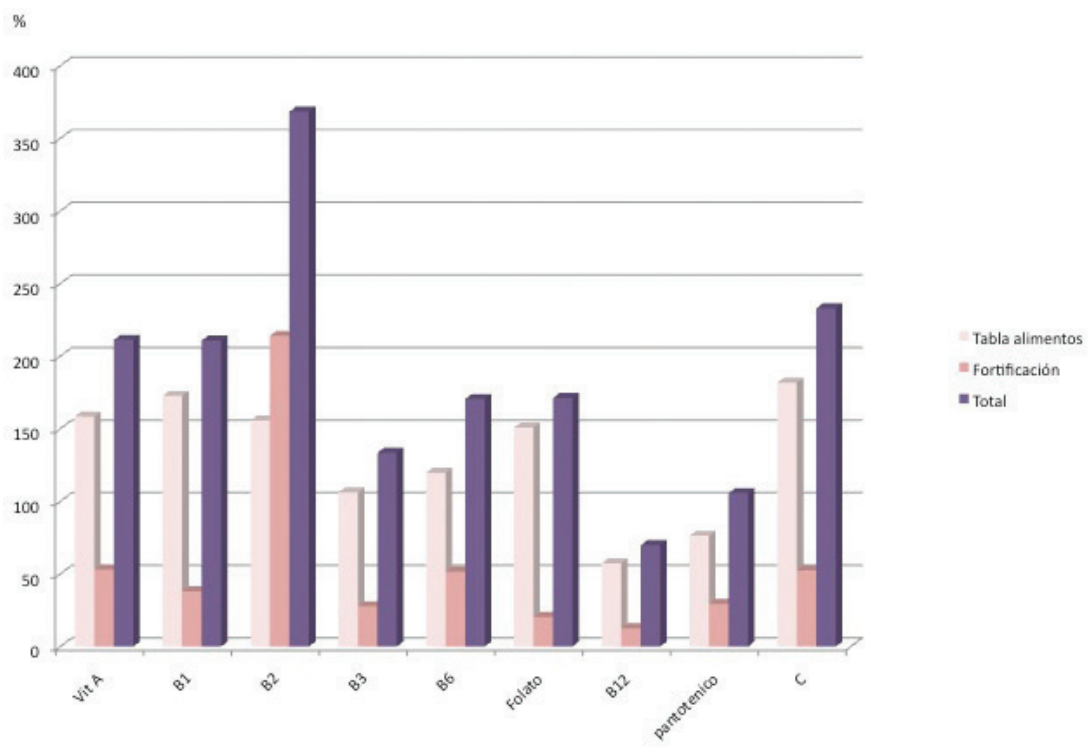


el riesgo podría incrementarse (19). Estudios europeos muestran una menor ingesta de folato al compararlo con nuestro estudio, que en el caso de las mujeres europeas era inferior a $2 / 3$ de la recomendación, el resto de las vitaminas del complejo B los resultados fueron similares (20). Una fuente importante de ácido fólico en nuestro país es el pan, estudios chilenos muestran que el consumo estimado en adolescentes de nivel socioeconómico bajo (NSB) de pan es de 250 g/día (21).

La única vitamina que estuvo bajo las recomendaciones fue la B12 en las estudiantes de sexo femenino, quizás por un consumo bajo de alimentos de origen animal o por llevar una dieta vegetariana con limitación o exclusión de alimentos de origen animal, en este último caso es necesario la utilización de suplementos con B12. Esta vitamina es un micronutriente esencial tanto para la maduración de los glóbulos rojos como en las múltiples funciones en diversas rutas metabólicas necesarias para la función del sistema nervioso central y periférico. La B12 juega un rol central en el metabolismo de un carbono, su déficit se ve a menudo en personas mayores, su deficiencia clínica se presenta con signos neurológicos anormales, pérdida de conocimiento, neuropatía periférica y trastornos psiquiátricos (22).

En nuestro estudio el porcentaje de estudiantes que están bajo las recomendaciones es bajo al compararlo con otros estudios (10), pero un número importante de ellos sobrepasa la UL para ciertas vitaminas. Estudios en Europa muestran que los niños son más propensos a superar la UL que en adultos especialmente con la vitamina $\mathrm{A}(16)$.

El 7,8\% de los escolares en nuestro estudio superó la UL para vitamina A, los efectos agudos y crónicos de esta vitamina están bien documentados, históricamente la toxicidad de vitamina A se ha asociado con alteraciones óseas (23-25) lo que se podría deber al antagonismo entre la vitamina A y D a nivel de receptor $(26,27)$ y a una interacción en la regulación calcio-hormonas (26). Evidencia reciente sugiere una subtoxicidad sin signos clínicos especialmente en países desarrollados, debido a que el consumo de fuentes de vitamina A preformada es superior a la ingesta recomendada. La osteoporosis y fracturas de cadera están asociadas a vitamina A preformada aún cuando el consumo solo duplique la recomendación (28).

La vitamina A se obtiene de la vitamina A preformada, es decir, esteres de retinol en alimentos de origen animal, alimentos fortificados y productos farmacéuticos, así como de provitamina A (carotenoides) de origen vegetal. La vitamina A preformada se absorbe y utiliza a tasas de absorción entre 70-90\% (29,30). En países industrializados la vitamina A preformada deriva de multivitaminas, aceite de pescado y la fortificación de alimentos como la leche, mantequilla, margarina, cereales para el desayuno y en algunos snacks. En países desarrollados estudios observacionales sugieren que más del $75 \%$ de la gente puede ingerir de forma rutinaria más de lo recomendado (31).

Nuestros resultados muestran un mayor porcentaje de sujetos que sobrepasa la UL al compararlo con un estudio norteamericano (10). Sin embargo la adecuada aplicación de valores de UL para niños es un tema controversial (32). La UL es el nivel más alto de consumo que no plantea riesgos de salud adversos (33). Pero aumentar el consumo sobre la UL el riesgo de efectos adversos puede incrementarse. Sin embargo la UL se basa en un enfoque de evaluación de riesgos y no se recomienda su utilización como una norma rígida o punto de corte, es necesario considerar otros factores que pueden tener efectos adversos para la salud (33). Se necesita más investigación sobre la salud, cuando el consumo supera la UL, debido a que la UL para los niños en varios nutrientes incluido el ácido fólico y la vitamina A se basan en datos con muchas limitaciones incluyendo la dosis respuesta y la toxicidad $(34,35)$.

Es necesario además determinar la UL para vitaminas como tiamina y rivoflavina, en este último caso la ingesta en nuestro estudio superaba el $300 \%$ de la recomendación.

Entre las fortalezas de nuestro estudio está la utilización de paneles con fotografías de los alimentos lo que permitió una mayor precisión al identificar el tipo y marca del alimento y entre las debilidades es la utilización de encuestas de tendencia de consumo las cuales tienden a sobreestimar las ingestas (36).

Se concluye que todas las vitaminas analizadas superan las recomendaciones a excepción de la B12 en mujeres. Otras vitaminas como folato, B3, B6 y vitamina A, superan la UL.

\section{RESUMEN}

Introducción: Las vitaminas son nutrientes que tienen múltiples beneficios para nuestro organismo, lo que hace que se utilicen para enriquecer alimentos con el fin de aumentar su aporte nutricional. Objetivo: Determinar si la ingesta de alimentos fortificados sobrepasa las recomendaciones dietarías (RDA) y el Nivel Máximo de Ingesta Tolerable (UL). Métodos: Se identificaron los 213 alimentos fortificados del mercado. Se aplicó una encuesta alimentaria utilizando un set fotográfico a 298 estudiantes secundarios de ambos sexos de la Región Metropolitana. Se calculó la ingesta de nutrientes y sobre el resultado se agregó las vitaminas fortificadas en los alimentos. Resultados: Los hombres superan la ingesta 
para todas las vitaminas, en cambio las mujeres presentan un déficit en la B12. Las vitaminas cuya ingesta supera la UL son, folato $27,5 \%$, B3 16,4\%, Vitamina A 7,8\% y B6 1,6\%. Conclusión: Todas las vitaminas analizadas superan las recomendaciones a excepción de la B12 en mujeres y vitaminas como folato, B3, B6 y vitamina A, superan la UL.

Palabras clave: vitaminas, encuesta alimentaria, UL, RDA.

Dirigir la correspondencia a:

Profesor

Samuel Durán Agüero

Universidad Autónoma de Chile, Santiago, Chile

Ricardo Morales 3369, San Miguel.

Santiago de Chile

E-mail: sduran74@gmail.com

Conflicto de interés: el presente estudio no presenta conflicto de intereses.

Agradecimientos: Agradecemos el apoyo entregado por las autoridades de la Universidad Autónoma de Chile, especialmente por la Facultad de Salud y Dirección de Carrera.

Destacamos el apoyo dado por el Departamento de Postgrado e Investigaciones, quienes financiaron el proyecto.

Además agradecemos a los alumnos de quinto año de la carrera de nutrición y Dietética: Ann Correa, Natalia Barros, Pamela Echeverría y Roberto del Río, por su valiosa colaboración en la confección y aplicación de encuestas y en la tabulación de los datos.

\section{BIBLIOGRAFÍA}

1. Herrán O, Prada G, Quintero D. Ingesta usual de vitaminas y minerales en Bucaramanga, Colombia. Rev Chil Nutr 2007;34:35-44.

2. Bowman BA, Russell RM (Editores). Conocimientos actuales sobre nutrición. 8 ed. ILSI. OPS/ OMS. Washington, Publicación científica y técnica número 592. 2003: p 723-61.

3. Nordic Council of Ministers. Integrating nutrition and physical activity. 4th ed. Copenhagen: Author; 2004. Nordic nutrition recommendations 2004.

4. Aghdessi E. Oxidative stress in smokers supplemented with vitamin C. Int J Vitamin Nutr Res 1999;69(1):45-51.

5. Bouchard $\mathrm{C}$. The magnitude of the energy imbalance in obesity is generally underestimated. Int J Obes (Lond) 2008;32:879-80.

6. Butte NF, Christiansen E, Sorensen TIA. Energy imbalance underlying the development of childhood obesity. Obesity (Silver Spring) 2007;15:3056-66.

7. Swinburn BA, Jolley D, Kremer PJ, Salbe AD, Ravussin E. Estimating the effects of energy imbalance on changes in body weight in children. Am J Clin Nutr 2006;83:859-63.

8. Wang YC, Gortmaker SL, Sobol AM, Kuntz KM. Estimating the energy gap among US children: a counterfactual approach. Pediatrics 2006;118:172133.

9. Reglamento Sanitario de los alimentos http./ www minsal gob cl/portal/wrl/page/minsalcl/g proteccion/g_alimentos/reglamento_sanitario_alimentos.html

10. Fulgoni VL, Keast DR, Bailey RL, Dwyer J. Foods, Fortificants, and Supplements' Where Do Americans Get Their Nutrients? I Nutr 2011;141:1847-54.

11. Bailey RL, Dodd KW, Gahche JJ, Dwyer JT, McDowell MA, Yetley EA, Sempos CA, Burt VL, Radimer KL, Picciano MF. Total folate and folic acid intake from foods and dietary supplements in the United States:2003-2006. Am J Clin Nutr 2010;91:231-7.

12. Bailey RL, Gahche JJ, Lentino CV, Dwyer JT, Engel JS, Thomas PR, Betz JM, Sempos CT, Picciano MF. Dietary supplement use in the United States, 2003-2006. J Nutr 2011;141:261-6.

13. Jury G, Urteaga C, Taibo M, Porciones de Intercambio y Composición Química de los Alimentos de la Pirámide Alimentaria Chilena. Santiago: Universidad de Chile, INTA; Facultad de Medicina, Centro de Nutrición Humana ,1999.

14. Nestlé. Tabla de composición química 2008. Santiago: Nestlé, 2008.

15. U.S. Department of Health and Human Services and USDA. Dietary Guidelines for Americans, 2010. 7th ed. Washington, DC: U.S. Government Printing Office; 2011.

16. Flynn A, Hirvonen T, Mensink GBM, Ocke MC, Serra-Majem L, Stos K, Szponar L, Tetens I, Turrini I, Fletcher I, et al. Intake of selected nutrients from foods, from fortification and from supplements in various European countries. Food Nutr Res $2009 \cdot 53 \cdot 1-51$

17. Hoey UcDulty H Askin N Bunne-A Ward M Pentieva K, Strain J, Molloy AM, Flynn CA, Scott $\mathrm{MM}$. Effect of a voluntary food fortification policy on folate, related B vitamin status, and homocysteine in healthy adults. Am J Clin Nutr 2007; 86:1405-13.

18. Hertrampf E, Cortés F. National food-fortification program with folic acid in Chile. Food Nutr Bul. 2008;29:231-7. 
19. Koren G. Folic acid and colorectal cancer: unwarranted fears. Can Fam Physician 2011;57:889-90.

20. J.Al-Tahan, M. González-Gross and K. Pietrzik BVitamin status and intake inEuropean adolescents. A review of the literatura. Nutr Hosp 2006;21:45265.

21. Olivares S, Albala C, García F, Jofré I. Publicidad televisiva y preferencias alimentarias en escolares de la Región Metropolitana. Rev Méd Chile 1999; 127:791-9.

22. Carmel R. How I treat cobalamin (vitamin B12) deficiency. Blood 2008;112: 2214-21.

23. Rohde CM, DeLuca H. Bone resorption activity of all-trans retinoic acid is independent of vitamin D in rats. J Nutr 2003;133:777- 83.

24. Binkley N, Krueger D. Hypervitaminosis A and bone. Nutr Rev 2000;58:138-44.

25. Johansson S, Melhus H. Vitamin A antagonizes calcium response to vitamin D in man. J Bone Miner Res 2001;16:1899-905.

26. Rohde CM, Manatt M, Clagett-Dame M, DeLuca $\mathrm{H}$. Vitamin A antagonizes the action of vitamin D in rats. J Nutr 1999;129:2246-50.

27. Aburto A, Edwards HM Jr, Britton WM. The influence of vitamin A on the utilization and amelioration of toxicity of cholecalciferol, 25-hydroxycholecalciferol, and 1,25 dihydroxycholecalciferol in Young broiler chickens. Poult Sci 1998;77:585-93.

28. Penniston K, Tanumihardjo S. The acute and chronic toxic effects of vitamin A. Am J Clin Nutr
2006;83:191-201.

29. Olson JA. Vitamin A. In: Ziegler EE, Filer LJ Jr, eds. Present knowledge in nutrition. 7th ed. Washington, DC: International Life Sciences Institute Press, 2001:p 109-119.

30. Mehta NJ. Dietary intervention with dark green leafy vegetables spinach (Spinacia oleracea) to combat subclinical vitamin A deficiency (SVAD) in slum children of Dharavi, Mumkbai, India. Sight Life Newslett 2001;4:32-33.

31. Allen LH, Haskell M. Estimating the potential for vitamin A toxicity in women and young children. J Nutr 2002;132:2907-19.

32. Berner LA, Levine MJ. Understanding tolerable upper intake levels: introduction to the workshop proceedings. J Nutr 2006;136:487-9.

33. Food and Nutrition Board, Institute of Medicine. Dietary reference intakes for thiamin, riboflavin, niacin, vitamin B-6, folate, vitamin B-12, pantothenic acid, biotin, and choline. Washington (DC): National Academies Press; 1998.

34. Renwick AG. Understanding tolerable upper intake levels: toxicology of micronutrients: adverse effects and uncertainties. J Nutr 2006;136:493-501.

35. Zlotkin S. Understanding tolerable upper intake levels: a critical assessment of the upper intake levels for infants and children. J Nutr 2006;136:502-6.

36. Urteaga $C$, Pinheiro AC. Investigación alimentaria: consideraciones practicas para mejorar la confiabilidad de los datos. Rev Chil Nutr 2003;30:235-2. 